PROCEEDINGS OF THE

AMERICAN MATHEMATICAL SOCIETY

Volume 137, Number 5, May 2009, Pages 1841-1847

S 0002-9939(08)09808-0

Article electronically published on December 29, 2008

\title{
TOPOLOGICAL COMPLEXITY OF CONFIGURATION SPACES
}

\author{
MICHAEL FARBER AND MARK GRANT
}

(Communicated by Alexander N. Dranishnikov)

\begin{abstract}
The topological complexity $\mathrm{TC}(X)$ is a homotopy invariant which reflects the complexity of the problem of constructing a motion planning algorithm in the space $X$, viewed as configuration space of a mechanical system. In this paper we complete the computation of the topological complexity of the configuration space of $n$ distinct points in Euclidean $m$-space for all $m \geq 2$ and $n \geq 2$; the answer was previously known in the cases $m=2$ and $m$ odd. We also give several useful general results concerning sharpness of upper bounds for the topological complexity.
\end{abstract}

\section{INTRODUCTION}

The motion planning problem is a central theme of robotics [14. Given a mechanical system $S$, a motion planning algorithm for $S$ is a function associating with any pair of states $(A, B)$ of $S$ a continuous motion of the system starting at $A$ and ending at $B$. If $X$ denotes the configuration space of the system, one considers the path fibration

$$
\pi: P X \rightarrow X \times X, \quad \pi(\gamma)=(\gamma(0), \gamma(1)),
$$

where $P X=X^{I}$ is the space of all continuous paths $\gamma: I=[0,1] \rightarrow X$. In these terms, a motion planning algorithm for $S$ is a section (not necessarily continuous) of $\pi$.

The topological complexity of a topological space $X$, denoted $\mathrm{TC}(X)$, is defined to be the genus, in the sense of Švarc [15, of fibration (1.1). More explicitly, TC $(X)$ is the minimal integer $k$ such that $X \times X$ admits a cover by $k$ open subsets, on each of which there exists a continuous local section of fibration (1.1). One of the basic properties of $\mathrm{TC}(X)$ is its homotopy invariance 6]. If $X$ is a Euclidean Neighbourhood Retract, then the number TC $(X)$ can be equivalently characterized (see [11, Proposition 4.2) as the minimal integer $k$ such that there exists a section $s: X \times X \rightarrow P X$ of (1.1) and a decomposition

$$
X \times X=G_{1} \cup \cdots \cup G_{k}, \quad G_{i} \cap G_{j}=\emptyset, \quad i \neq j,
$$

where each $G_{i}$ is locally compact and such that the restriction $s \mid G_{i}: G_{i} \rightarrow P X$ is continuous for $i=1, \ldots, k$. A section $s$ as above can be viewed as a motion planning algorithm: given a pair of states $(A, B) \in X \times X$, the path $s(A, B)(t)$

Received by the editors June 25, 2008.

2000 Mathematics Subject Classification. Primary 55M99, 55R80; Secondary 68T40.

Key words and phrases. Topological complexity, configuration spaces.

This research was supported by grants from the EPSRC and from The Royal Society.

(C)2008 American Mathematical Society 
represents a continuous motion of the system starting from $A$ and ending at $B$. The number $\mathrm{TC}(X)$ is a measure of the complexity of motion planning algorithms for a system whose configuration space is $X$.

The concept TC $(X)$ was introduced and studied in [6, 7]. We refer the reader to surveys [9, 11] for a detailed treatment of the invariant TC $(X)$. Computation of $\mathrm{TC}(X)$ in various practically interesting examples has received much recent interest; see for instance papers [1, 2], 10, [12, 13.

In this paper we study the topological complexity $\mathrm{TC}\left(F\left(\mathbf{R}^{m}, n\right)\right)$ of the space of configurations of $n$ distinct points in Euclidean $m$-space. Here $m, n \geq 2$, and

$$
F\left(\mathbf{R}^{m}, n\right)=\left\{\left(\mathrm{x}_{1}, \ldots, \mathrm{x}_{n}\right) \in\left(\mathbf{R}^{m}\right)^{\times n} ; \mathrm{x}_{i} \neq \mathrm{x}_{j} \text { for } i \neq j\right\},
$$

topologised, as a subspace of the Cartesian power $\left(\mathbf{R}^{m}\right)^{\times n}$. This space appears in robotics when one controls multiple objects simultaneously, trying to avoid collisions between them. Our main result in this paper is the following.

Theorem 1.1. One has

$$
\mathrm{TC}\left(F\left(\mathbf{R}^{m}, n\right)\right)= \begin{cases}2 n-1 & \text { for all } m \text { odd }, \\ 2 n-2 & \text { for all } m \text { even. }\end{cases}
$$

The cases $m=2$ and $m \geq 3$ odd of Theorem 1.1 were proven by Farber and Yuzvinsky in [8], where it was conjectured that $\operatorname{TC}\left(F\left(\mathbf{R}^{m}, n\right)\right)=2 n-2$ for all even $m$. Here we settle this conjecture in the affirmative. Note that the methods employed in [8] are not applicable in the case when $m>2$ is even. We therefore suggest an alternative approach based on sharp upper bounds for the topological complexity.

The plan of the paper is as follows. In the next section we state Theorems 2.1 and 2.2 about sharp upper bounds; their proofs appear in section 3. The concluding section 4 contains the proof of Theorem 1.1

\section{Sharp UPPER BOUNDS FOR THE TOPOLOGICAL COMPLEXITY}

Let $X$ be a CW-complex of finite dimension $\operatorname{dim}(X)=n \geq 1$. We denote by $\Delta_{X} \subset X \times X$ the diagonal $\Delta_{X}=\{(\mathrm{x}, \mathrm{x}) ; \mathrm{x} \in X\}$. Let $A$ be a local system of coefficients on $X \times X$. A cohomology class

$$
u \in H^{*}(X \times X ; A)
$$

is called a zero-divisor if its restriction to the diagonal is trivial, i.e. $u \mid \Delta_{X}=0 \in$ $H^{*}(X ; A \mid X)$. The importance of zero-divisors stems from the following fact (see [11], Corollary 4.40):

If the cup-product of $k$ zero-divisors $u_{i} \in H^{*}\left(X \times X ; A_{i}\right)$, where $i=1, \ldots, k$, is nonzero, then $\mathrm{TC}(X)>k$.

Theorem 2.1 below supplements the general dimensional upper bound of $[6$ by giving necessary and sufficient conditions for its sharpness.

Theorem 2.1. For any $n$-dimensional cell complex $X$ one has

(a) $\mathrm{TC}(X) \leq 2 n+1$;

(b) $\mathrm{TC}(X)=2 n+1$ if and only if there exists a local coefficient system $A$ on $X \times X$ and a zero-divisor $\xi \in H^{1}(X \times X ; A)$ such that the $2 n$-fold cup product

$$
\xi^{2 n}=\xi \cup \cdots \cup \xi \neq 0 \in H^{2 n}\left(X \times X ; A^{2 n}\right)
$$


is nonzero. Here $A^{2 n}$ denotes the tensor product of $2 n$ copies $A \otimes \cdots \otimes A$ of $A$ (over $\mathbf{Z}$ ).

Next we state a similar sharp upper bound result for $(s-1)$-connected spaces $X$ where $s>1$. We use the following notation. If $B$ is an abelian group and $v \in H^{r}(X ; B)$ is a cohomology class, then the class

$$
\bar{v}=v \times 1-1 \times v \in H^{r}(X \times X ; B)
$$

is a zero-divisor, where $1 \in H^{0}(X ; \mathbf{Z})$ is the unit and $\times$ denotes the cohomological cross-product.

We say that a finitely generated abelian group is square-free if it has no subgroups isomorphic to $\mathbf{Z}_{p^{2}}$, where $p$ is a prime.

Theorem 2.2. Let $X$ be an $(s-1)$-connected $n$-dimensional finite cell complex where $s \geq 2$. Assume additionally that $2 n=r s$ where $r$ is an integer 1 Then

(a) $\mathrm{TC}(X) \leq r+1$.

(b) $\mathrm{TC}(X)=r+1$ if and only if there exists a finitely generated abelian group $B$ and a cohomology class $v \in H^{s}(X ; B)$ such that the $n$-fold cup-product of the corresponding zero-divisors (2.1) is nonzero,

$$
\bar{v}^{r}=\bar{v} \cup \cdots \cup \bar{v} \neq 0 \in H^{2 n}\left(X \times X ; B^{r}\right) .
$$

Here $B^{r}$ denotes the $r$-fold tensor power $B \otimes \cdots \otimes B$.

(c) If $H_{*}(X ; \mathbf{Z})$ is square-free, then $\mathrm{TC}(X)=r+1$ if and only if there exists a field $\mathbf{k}$ and cohomology classes $v_{1}, \ldots, v_{r} \in H^{s}(X ; \mathbf{k})$ such that

$$
\bar{v}_{1} \cup \cdots \cup \bar{v}_{r} \neq 0 \in H^{2 n}(X \times X ; \mathbf{k}) .
$$

(d) If $H_{s}(X ; \mathbf{Z})$ is free abelian, then $\mathrm{TC}(X)=r+1$ if and only if there exist classes $v_{1}, \ldots, v_{r} \in H^{s}(X ; \mathbf{Z})$ such that

$$
\bar{v}_{1} \cup \cdots \cup \bar{v}_{r} \neq 0 \in H^{2 n}(X \times X ; \mathbf{Z}) .
$$

\section{Proofs of Theorems 2.1 and 2.2}

Proof of Theorem 2.1. The first statement follows from [6, Theorem 4. If there exists a local coefficient system $A$ and a zero-divisor $\xi \in H^{1}(X \times X ; A)$ such that $\xi^{2 n} \neq 0$, then $\mathrm{TC}(X) \geq 2 n+1$, by Corollary 4.40 of [11. The remaining part of Theorem 2.1 was proven in [3], Theorem 7. More precisely, let $G=\pi_{1}\left(X, x_{0}\right)$ denote the fundamental group of $X$ and let $I \subset \mathbf{Z}[G]$ denote the augmentation ideal. $I$ can be viewed as a left $\mathbf{Z}[G \times G]$-module via the action

$$
(g, h) \cdot \sum n_{i} g_{i}=\sum g g_{i} h^{-1},
$$

where $g, h \in G$ and $\sum n_{i} g_{i} \in I$. This defines a local system with stem $I$ on $X \times X$; see [16], chapter 6 . A crossed homomorphism $f: G \times G \rightarrow I$ given by the formula

$$
f(g, h)=g h^{-1}-1, \quad g, h \in G
$$

determines a cohomology class $\mathfrak{v} \in H^{1}(X \times X ; I)$. This class is a zero-divisor and has the property that $\mathfrak{v}^{2 n} \neq 0$ assuming that $\mathrm{TC}(X)=2 n+1$ according to Theorem 7 from 3 .

\footnotetext{
${ }^{1}$ This last assumption is automatically satisfied (with $r=n$ ) for $s=2$, i.e. when $X$ is simply connected.
} 
Proof of Theorem 2.2. Statement (a) follows directly from Theorem 5.2 of [7, which states that

$$
\mathrm{TC}(X)<\frac{2 n+1}{s}+1
$$

for any $(s-1)$-connected CW-complex $X$ of dimension $n$.

(b) One part of statement (b) follows from Corollary 4.40 of [11]; indeed if $\bar{v}^{r} \neq 0$, then $\mathrm{TC}(X) \geq r+1$ since each $\bar{v}$ is a zero-divisor.

The proof of the remaining part of statement (b) is derived from obstruction theory and results of A. S. Švarc [15] centered around the notion of genus of a fibration. We assume that $X$ is $(s-1)$-connected, $s \geq 2$, and $n$-dimensional and $2 n=r s$, where $r$ is an integer. The case $n=1$ is trivial; therefore we will assume that $n \geq 2$. We want to show that $\mathrm{TC}(X)=r+1$ implies that $\bar{v}^{r} \neq 0 \in H^{2 n}(X \times$ $\left.X ; B^{r}\right)$ for some class $v \in H^{s}(X ; B)$.

Recall that TC $(X)$ is defined as the genus of the path fibration (1.1) and according to Theorem 3 from [15] one has $\mathrm{TC}(X) \leq r$ if and only if the $r$-fold fiberwise join

$$
\pi_{r}: P_{r} X \rightarrow X \times X
$$

of the original fibration $\pi: P X \rightarrow X \times X$ admits a continuous section. Hence our assumption $\mathrm{TC}(X)=r+1$ implies that $\pi_{r}$ has no continuous sections. The fibre $F_{r}$ of (3.2) is the $r$-fold join

$$
F_{r}=\Omega X * \Omega X * \cdots * \Omega X
$$

where $\Omega X$ denotes the space of loops in $X$ starting and ending at the base point $x_{0} \in X$. Note that $\Omega X$ is $(s-2)$-connected and therefore the fibre $F_{r}$ is $(2 n-2)$ connected since $2 r(s-2)+2(r-1)=2 n-2$.

The primary obstruction to the existence of a section of (3.2) is an element $\theta_{r} \in H^{2 n}\left(X \times X ; \pi_{2 n-1}\left(F_{r}\right)\right)$. It is in fact the only obstruction since the higher obstructions land in zero groups. Thus we obtain that $\theta_{r} \neq 0$. By the Hurewicz theorem

$$
\pi_{2 n-1}\left(F_{r}\right)=H_{2 n-1}\left(F_{r}\right)=B \otimes B \otimes \cdots \otimes B=B^{r},
$$

where $B$ denotes the abelian group $H_{s-1}(\Omega X)=H_{s}(X)$. Here we have used the Künneth theorem for joins; see for instance [15], chapter $1, \S 5$. By Theorem 1 from [15] the obstruction $\theta_{r}$ equals the $r$-fold cup-product

$$
\theta_{r}=\theta \cup \cdots \cup \theta=\theta^{r},
$$

where $\theta \in H^{s}(X \times X ; B)$ is the primary obstruction to the existence of a section of $\pi: P X \rightarrow X \times X$. Writing $\theta=v \times 1+1 \times w$ and observing that $\theta \mid \Delta_{X}=0$ (since there is a continuous section of (1.1) over the diagonal $\Delta_{X} \subset X \times X$ ) shows that $v+w=0$ and therefore $\theta=v \times 1-1 \times v=\bar{v}$. Hence we have found a cohomology class $v \in H^{s}(X ; B)$ with $\bar{v}^{r} \neq 0$.

(c) In one direction the statement of (c) follows from the upper bound (a) and [6. Thm. 7]; i.e. the existence of classes $v_{1}, \ldots, v_{r} \in H^{s}(X ; \mathbf{k})$ with $\bar{v}_{1} \cup \cdots \cup \bar{v}_{r} \neq 0$

\footnotetext{
${ }^{2}$ One knows that the join of a $p$-connected complex and a $q$-connected complex is $(p+q+2)$ connected.
} 
combined with (a) gives $\mathrm{TC}(X)=r+1$. Suppose now that $H_{*}(X)$ is square-free. Write $B=H_{s}(X)$ as a direct sum

$$
B=\bigoplus_{i \in I} B_{i}
$$

where each $B_{i}$ is either $\mathbf{Z}$ or a cyclic group of prime order $\mathbf{Z}_{p}$ and $I$ is an index set. The $r$-fold tensor power $B^{r}=B \otimes \cdots \otimes B$ is a direct sum

$$
B^{r}=\bigoplus_{\left(i_{1}, \ldots, i_{r}\right) \in I^{r}} B_{i_{1}} \otimes B_{i_{2}} \otimes \cdots \otimes B_{i_{r}}
$$

and each tensor product $B_{i_{1}} \otimes B_{i_{2}} \otimes \cdots \otimes B_{i_{r}}$ is either $\mathbf{Z}, \mathbf{Z}_{p}$ or trivial. As we know from the proof of (b) there is a class $v \in H^{s}(X ; B)$ such that $\bar{v}^{r} \neq 0 \in$ $H^{2 n}\left(X \times X ; B^{r}\right)$. For any index $i \in I$ denote by $v_{i}$ the image of $v$ under the coefficient projection $B \rightarrow B_{i}$. Since $\bar{v}^{r} \neq 0$ there exists a sequence $\left(i_{1}, \ldots, i_{r}\right) \in I^{r}$ such that the product

$$
\bar{v}_{i_{1}} \cup \cdots \cup \bar{v}_{i_{r}} \in H^{2 n}\left(X \times X ; B_{i_{1}} \otimes B_{i_{2}} \otimes \cdots \otimes B_{i_{r}}\right)
$$

is nonzero. If the product $B_{i_{1}} \otimes B_{i_{2}} \otimes \cdots \otimes B_{i_{r}}$ is $\mathbf{Z}_{p}$, then each $B_{i_{j}}$ is either $\mathbf{Z}$ or $\mathbf{Z}_{p}$ and taking $\mathbf{k}=\mathbf{Z}_{p}$ and reducing all these classes $v_{i_{k}} \bmod p$ we obtain that (c) is satisfied. In the case when the product $B_{i_{1}} \otimes B_{i_{2}} \otimes \cdots \otimes B_{i_{r}}$ is infinite cyclic each of the groups $B_{i_{k}}$ is $\mathbf{Z}$ and the class

$$
\bar{v}_{i_{1}} \cup \cdots \cup \bar{v}_{i_{r}} \neq 0 \in H^{2 n}(X \times X ; \mathbf{Z})
$$

is integral and nonzero.

Since the group $H^{2 n}(X \times X ; \mathbf{Z})$ is square-free the cup-product (3.4) is indivisible by some prime $p$. Indeed, the group $H^{2 n}(X \times X ; \mathbf{Z})$ is a direct sum of cyclic groups of prime order and infinite cyclic groups and the product (3.4) has a nontrivial component in at least one of these groups. A nonzero element of $\mathbf{Z}$ is divisible by finitely many primes, and a nonzero element of $\mathbf{Z}_{p}$ is divisible by all primes except $p$.

Therefore, as follows from the exact sequence

$$
\cdots \rightarrow H^{2 n}(X \times X ; \mathbf{Z}) \stackrel{p}{\rightarrow} H^{2 n}(X \times X ; \mathbf{Z}) \rightarrow H^{2 n}\left(X \times X ; \mathbf{Z}_{p}\right) \rightarrow \ldots,
$$

the $\bmod p$ reduction of the product (3.4) is nonzero. Now, taking $\mathbf{k}=\mathbf{Z}_{p}$ and reducing the classes $v_{i_{k}}$ mod $p$, we get a sequence of classes $w_{j_{k}} \in H^{s}(X ; \mathbf{k})$ such that $\prod \bar{w}_{j_{k}} \neq 0$, where $k=1, \ldots, r$.

(d) The proof of statement (d) of Theorem 2.2 is similar to that of (c), with the simplification that all the groups $B_{i}$ are in this case infinite cyclic.

\section{Proof of Theorem 1}

The cases $m=2$ and $m \geq 3$ odd of Theorem 1 were dealt with by Farber and Yuzvinsky in [8]. Their arguments also show that if $m \geq 4$ is even, then $\mathrm{TC}\left(F\left(\mathbf{R}^{m}, n\right)\right)$ equals either $2 n-1$ or $2 n-2$. Hence to prove Theorem 1 it suffices to show that $\operatorname{TC}\left(F\left(\mathbf{R}^{m}, n\right)\right) \neq 2 n-1$ when $m \geq 4$ is even.

Fix $n \geq 2$. For any $m \geq 2$ the space $F\left(\mathbf{R}^{m}, n\right)$ is $(m-2)$-connected, since it is the complement of an arrangement of codimension $m$ subspaces of $\mathbf{R}^{m n}$. Its integral cohomology ring is shown in [5] to be a graded commutative algebra over $\mathbf{Z}$ on generators

$$
e_{i j} \in H^{m-1}\left(F\left(\mathbf{R}^{m}, n\right)\right), \quad 1 \leq i<j \leq n,
$$


subject to the relations

$$
e_{i j}^{2}=0, \quad e_{i j} e_{i k}=\left(e_{i j}-e_{i k}\right) e_{j k}
$$

for any triple $1 \leq i<j<k \leq n$. In particular, $H^{*}\left(F\left(\mathbf{R}^{m}, n\right)\right)$ is nonzero only in dimensions $i(m-1)$, where $i=0,1, \ldots,(n-1)$. Applying the result of Eilenberg and Ganea [4] we obtain that for $m \geq 3$ the space $F\left(\mathbf{R}^{m}, n\right)$ is homotopy equivalent to a finite complex of dimension $\leq(m-1)(n-1)$. Now we may apply statement (d) of Theorem 2.2, which gives, firstly, that $\mathrm{TC}\left(F\left(\mathbf{R}^{m}, n\right)\right) \leq 2 n-1$ and, secondly, $\mathrm{TC}\left(F\left(\mathbf{R}^{m}, n\right)\right)=2 n-1$ if and only if there exist cohomology classes

$$
v_{1}, \ldots, v_{2(n-1)} \in H^{m-1}\left(F\left(\mathbf{R}^{m}, n\right)\right)
$$

such that the product of the corresponding zero-divisors

$$
\bar{v}_{1} \cup \bar{v}_{2} \cup \cdots \cup \bar{v}_{2(n-1)}
$$

is nonzero; recall that the notation $\bar{v}$ is introduced in (2.1). We show below that such classes $v_{1}, \ldots, v_{2(n-1)}$ do not exist if $m \geq 4$ is even.

We recall the result of $[8$ stating that $\operatorname{TC}(F(\mathbf{C}, n))=2 n-2$. It is shown in the proof of Theorem 6 in $[8$ ] that $F(\mathbf{C}, n)$ is homotopy equivalent to the product $X \times S^{1}$, where $X$ is a finite polyhedron of dimension $\leq n-2$. This argument uses the algebraic structure of $\mathbf{C}=\mathbf{R}^{2}$ and does not generalize to $F\left(\mathbf{R}^{m}, n\right)$ with $m>2$. Using the product inequality (Theorem 11 in [6]) one obtains

$$
\begin{aligned}
\mathrm{TC}(F(\mathbf{C}, n)) & \leq \mathrm{TC}(X)+\mathrm{TC}\left(S^{1}\right)-1 \\
\leq & (2(n-2)+1)+2-1=2 n-2 .
\end{aligned}
$$

Hence there exist no $2(n-1)$ cohomology classes $v_{1}, \ldots, v_{2(n-1)} \in H^{1}(F(\mathbf{C}, n))$ such that the product of the zero-divisors $\bar{v}_{1} \cup \cdots \cup \bar{v}_{2(n-1)}$ is nonzero, as this would contradict Theorem 7 from 6 .

Now we observe that for any even $m \geq 2$ there is an algebra isomorphism

$$
\phi: H^{*}(F(\mathbf{C} ; n)) \rightarrow H^{*(m-1)}\left(F\left(\mathbf{R}^{m}, n\right)\right)
$$

mapping classes of degree $i$ to classes of degree $(m-1) i$, where $i=0,1, \ldots, n-1$; see [5]. Thus we conclude that there exist no cohomology classes $w_{1}, \ldots, w_{2(n-1)} \in$ $H^{m-1}\left(F\left(\mathbf{R}^{m}, n\right)\right)$ such that the product of the corresponding zero-divisors $\bar{w}_{1} \cup \cdots \cup$ $\bar{w}_{2(n-1)}$ is nonzero. Theorem 2.2 (statement $\left.(\mathrm{d})\right)$ now gives that $\mathrm{TC}\left(F\left(\mathbf{R}^{m}, n\right)\right) \leq$ $2(n-1)$.

On the other hand, it is proven in 8 ] that one may find $2 n-3$ cohomology classes $v_{1}, \ldots, v_{2 n-3} \in H^{1}(F(\mathbf{C}, n))$ such that the cup-product $\bar{v}_{1} \cup \cdots \cup \bar{v}_{2 n-3}$ is nonzero. Hence, repeating the above argument we see that for $m$ even there exist classes $w_{1}, \ldots, w_{2 n-3} \in H^{m-1}\left(F\left(\mathbf{R}^{m}, n\right)\right)$ (where $w_{i}=\phi\left(v_{i}\right)$ ) with nonzero product $\bar{w}_{1} \cup \cdots \cup \bar{w}_{2 n-3}$; this gives the opposite inequality $\operatorname{TC}\left(F\left(\mathbf{R}^{m}, n\right)\right) \geq 2 n-2$.

Hence, $\operatorname{TC}\left(F\left(\mathbf{R}^{m}, n\right)\right)=2 n-2$ as stated.

\section{REFERENCES}

[1] D. C. Cohen and G. Pruidze, Motion planning in tori, Bull. London Math. Soc. 40 (2008), 249-262. MR2414784

[2] D. C. Cohen and G. Pruidze, Topological complexity of basis-conjugating automorphism groups, preprint. [arXiv:0804.1825]

[3] A. Costa and M. Farber, Motion planning in spaces with "small" fundamental groups, preprint, 2008.

[4] S. Eilenberg and T. Ganea, On the Lusternik-Schnirelmann category of abstract groups, Ann. of Math. (2) 65 (1957), 517-518. MR0085510(19:52d) 
[5] E. R. Fadell and S. Y. Husseini, Geometry and Topology of Configuration Spaces, Springer Monographs in Mathematics, Springer-Verlag, Berlin, 2001. MR1802644 (2002k:55038)

[6] M. Farber, Topological complexity of motion planning, Discrete Comput. Geom. 29 (2003), 211-221. MR1957228 (2004c:68132)

[7] M. Farber, Instabilities of robot motion, Topology Appl. 140 (2004), 245-266. MR 2074919 (2005g:68166)

[8] M. Farber and S. Yuzvinsky, Topological robotics: Subspace arrangements and collision free motion planning, In "Geometry, Topology, and Mathematical Physics", Amer. Math. Soc. Transl. (2) 212, Amer. Math. Soc., Providence, RI, 2004, 145-156. MR2070052 (2005i:55019)

[9] M. Farber, Topology of robot motion planning, in: "Morse Theoretic Methods in Nonlinear Analysis and in Symplectic Topology" (P. Biran et al. (eds.)), Springer, Dordrecht, 2006, 185-230. MR2276952 (2008d:68141)

[10] M. Farber and M. Grant, Robot motion planning, weights of cohomology classes, and cohomology operations, Proc. Amer. Math. Soc. 136 (2008), 3339-3349. MR2407101

[11] M. Farber, Invitation to Topological Robotics, EMS, 2008.

[12] J. González, Topological robotics in lens spaces, Math. Proc. Cambridge Philos. Soc. 139 (2005), no. 3, 469-485. MR2177172 (2006f:55006)

[13] M. Grant, Topological complexity of motion planning and Massey products, to appear in Proceedings of the M. M. Postnikov Memorial Conference 2007, Banach Centre Publications [arxiv:0709.2287].

[14] J.-C. Latombe, Robot Motion Planning, Kluwer, Dordrecht, 1991.

[15] A. S. Švarc, The genus of a fiber space, Amer. Math. Soc. Transl. (2) 55, Amer. Math. Soc., Providence, RI, 1966, 49-140.

[16] G.W. Whitehead, Elements of Homotopy Theory, Springer-Verlag, New York, 1978. MR516508(80b:55001)

Department of Mathematical Sciences, Durham University, South Road, Durham, DH1 3LE, United Kingdom

E-mail address: michael.farber@durham.ac.uk

School of Mathematics, The University of Edinburgh, King's Buildings, Edinburgh, EH9 3JZ, United Kingdom

E-mail address: mark.grant@ed.ac.uk 